\title{
Association between the $E G F$ rs4444903 polymorphism and liver cancer susceptibility: a meta-analysis and meta-regression
}

\author{
Y.L. $\mathrm{Li}^{1}$, Z. $\operatorname{Tian}^{2}$, L. Zhao ${ }^{2}$ and C.L. Zhang ${ }^{2}$ \\ ${ }^{1}$ Department of Gastroenterology, \\ The First Affiliated Hospital of China Medical University, Shenyang, China \\ ${ }^{2}$ Department of General Surgery, \\ The Shengjing Affiliated Hospital of China Medical University, Shenyang, China \\ Corresponding author: Y.L. Li \\ E-mail: 1yl-72@163.com
}

Genet. Mol. Res. 13 (4): 8066-8079 (2014)

Received June 10, 2013

Accepted June 10, 2014

Published October 7, 2014

DOI http://dx.doi.org/10.4238/2014.October.7.1

\begin{abstract}
Emerging evidence suggests that a common functional polymorphism, rs4444903 (A>G), in the $E G F$ gene might impact an individual's susceptibility to liver cancer; however, individually published results are inconclusive. This meta-analysis aimed to derive a more precise estimation of the relationship between the $E G F$ rs4444903 polymorphism and liver cancer risk. A literature search was conducted in the PubMed, Embase, Web of Science, and CBM databases from inception through May 1st, 2013. Seven case-control studies were included with a total of 1408 liver cancer cases and 1343 healthy controls. Crude odds ratios (ORs) with 95\% confidence intervals (CIs) were calculated. Our meta-analysis results indicated that the $\mathrm{G}$ variant of the rs 4444903 polymorphism might be associated with an increased risk of liver cancer ( $\mathrm{G}$ allele $v s$ A allele: $\mathrm{OR}=1.25$, $95 \% \mathrm{CI}=1.01-1.56, \mathrm{P}=0.040 ; \mathrm{GG}+\mathrm{AG} v s \mathrm{AA}: \mathrm{OR}=1.65,95 \% \mathrm{CI}=$ $1.27-2.15, \mathrm{P}<0.001 ; \mathrm{GG} v \mathrm{AA}: \mathrm{OR}=1.77,95 \% \mathrm{CI}=1.34-2.35, \mathrm{P}<$ $0.001)$. Further subgroup analysis by ethnicity also showed significant
\end{abstract}


associations between the $\mathrm{G}$ variant of the rs 4444903 polymorphism and an increased risk of liver cancer among Asian, Caucasian, and African populations. No publication bias was detected in this meta-analysis. In conclusion, the current meta-analysis suggests that the $G$ variant of the rs4444903 polymorphism may increase the risk of liver cancer. The $E G F$ rs4444903 (A>G) polymorphism can be useful as a biomarker in predicting the development of liver cancer.

Key words: EGF; Polymorphism; Liver cancer; Meta-analysis; Meta-regression

\section{INTRODUCTION}

Liver cancer is both the sixth most common cancer throughout the world and the third most common cause of cancer mortality (Kirk et al., 2006; Chuang et al., 2009), and the global incidence of liver cancer is still increasing (Villanueva et al., 2012). Several important risk factors have been demonstrated in liver cancer, including environmental risks and genetic factors. The risk factors established for liver cancer include hepatitis viruses (HBV and HCV), alcohol consumption, aflatoxin, iron overload, geographic distribution, and some inherited genes (Srivatanakul et al., 2004; Hussain et al., 2007; Altekruse et al., 2012). A growing understanding on the molecular pathogenesis of liver cancer has demonstrated the association between genetic changes and liver cancer. Mutations in some genes have been shown to increase the risk of liver cancer, such as COX-2, LAPTM4B, ERCC1, p53, CYP2E1, and EGF (Kato et al., 2003; Kovar et al., 2009; Hu et al., 2010; Chen et al., 2011; He et al., 2011; Wang et al., 2012).

The human $E G F$ gene, encoding epidermal growth factor, is located on chromosome 4q25-q27 and has been reported to be associated with liver cancer susceptibility (Wu et al., 2009; Yotsumoto et al., 2009; Araujo et al., 2011; Daraei et al., 2012; de Mello et al., 2012). Mutational and functional analyses support the idea that the most common polymorphism rs4444903 $(\mathrm{A}>\mathrm{G})$, located in the 5' untranslated region at position 61 in the $E G F$ gene, might influence the expression level of EGF and was associated with the risk of liver cancer (Tanabe et al., 2008). Most previous studies support the hypothesis that $E G F$ gene mutations promote the development of liver cancer (Xu et al., 2010; Yang et al., 2012). However, there are also some studies suggesting no association between $E G F$ gene mutations and susceptibility to liver cancer (Qi et al., 2009). In view of the conflicting results of previous studies and the insufficient statistical power of a single study, we performed a meta-analysis of all case-control studies to further evaluate the precise association between the $E G F$ rs4444903 (A>G) polymorphism and liver cancer risk.

\section{MATERIAL AND METHODS}

\section{Literature search}

A comprehensive search for relevant studies was conducted in the PubMed, Embase, Web of Science, and CBM databases from inception through May 1st, 2013, using the following terms: ("Genetic polymorphism" or "polymorphism" or "SNP" or "gene mutation" or "ge- 
netic variants") and ("liver neoplasms" or "hepatic neoplasms" or "liver cancer" or "hepatic cancer" or "hepatocellular carcinoma" or "hepatocellular cancer") and ("epidermal growth factor" or " $E G F$ " or "human epidermal growth factor"). There were no language restrictions. The references used in eligible articles or textbooks were also reviewed to find other potential studies. Any disagreements were resolved by discussions and consensus.

\section{Inclusion and exclusion criteria}

Studies included in our meta-analysis had to meet the following criteria: 1) casecontrol studies focused on the association between the $E G F$ rs4444903 (A>G) polymorphism and liver cancer susceptibility; 2) all patients with the diagnosis of hepatic cancer should be confirmed by pathological examinations; 3 ) the genotype distribution of the controls should conform to Hardy-Weinberg equilibrium (HWE); 4) published data about the allele and genotype frequencies of SNPs must be sufficient. Studies were excluded when they were: 1) not a case-control study; 2) duplicate publications of data from the same study; 3) based on incomplete data; 4) deviated from HWE in the genotype frequencies of the controls; 5) metaanalyses, letters, reviews, or editorial articles. If more than one study by the same author using the same case series was published, either the study with the largest sample size or the most recent publication was included.

\section{Data extraction}

Data from the studies published were extracted independently by two authors into a standardized form. For each study, the following characteristics and numbers were collected: the first author, year of publication, country, language, ethnicity of subjects, study design, number of subjects, source of cases and controls, detecting sample, genotyping method, allele and genotype frequencies, and evidence of HWE in controls. In cases of conflicting evaluations, disagreements on inconsistent data from the eligible studies were resolved through discussions and careful reexamination of the full text by the authors.

\section{Quality assessment of studies included}

Two authors independently assessed the quality of papers according to the modified STROBE quality scoring system (da Costa et al., 2011). Forty assessment items related to quality appraisal were used in this meta-analysis with scores ranging from 0 to 40 . On the basis of their scores, the studies included were classified into three levels: low quality (0-19), moderate quality (20-29), and high quality (30-40), respectively. Disagreements on STROBE scores of the included studies were resolved through a comprehensive reassessment by the authors.

\section{Statistical analysis}

Crude odds ratios (ORs) with 95\% confidence intervals (CIs) were calculated under five genetic models: allele model ( $\mathrm{G}$ allele $v s \mathrm{C}$ allele), dominant model ( $\mathrm{GG}+\mathrm{AG} v s \mathrm{AA}$ ), recessive model (GG $v s \mathrm{AA}+\mathrm{AG})$, homozygous model (GG $v s \mathrm{AA})$, and heterozygous model 
(GG vs AG). Genotype frequencies of controls were tested for HWE using the $\chi^{2}$ test for each study included in the meta-analysis. The statistical significance of the pooled OR was examined using the $Z$ test. Between-study heterogeneity was estimated using Cochran's $Q$ statistic, whereas a $\mathrm{P}_{\mathrm{h}}<0.05$ was set to identify heterogeneity in the associations (Higgins and Thompson, 2002). We also quantified the effects of heterogeneity using the $I^{2}$ test (ranges from 0 to $100 \%$ ), which represents the proportion of inter-study variability that is contributed by heterogeneity rather than by chance (Zintzaras and Ioannidis, 2005). When a significant $Q$-test $(\mathrm{P}<0.05)$ or $I^{2}>50 \%$ indicated heterogeneity among studies, the random effects model (DerSimonian Laird method) was conducted for meta-analysis. Otherwise, the fixed effects model (Mantel-Haenszel method) was used. To explore the potential sources of heterogeneity, subgroup analysis was performed by ethnicity, country, source of controls, language, and detection sample. Univariate and multivariate regression analyses were also performed to identify the contributions of different variables to the heterogeneity of the associations (Ioannidis et al., 2008). Sensitivity analysis was performed by omitting each study in turn to assess the quality and consistency of the results. Begg's funnel plots and Egger's linear regression test were used to evaluate the publication bias (Peters et al., 2006). Two-sided $\mathrm{P}<0.05$ was considered to be statistically significant. All calculations were performed using the STATA version 12.0 software (STATA Corporation; College Station, TX, USA).

\section{RESULTS}

\section{Characteristics of studies included}

In accordance with the inclusion criteria, seven case-control studies (Tanabe et al., 2008; Qi et al., 2008, 2009; Wang, 2009; Li et al., 2010; Chen et al., 2011; Abbas et al., 2012) were included and 38 articles were excluded. The flow chart of the study selection process is shown in Figure 1. The publication year of studies included ranged from 2008 to 2012. A total of 2751 subjects were involved in this meta-analysis, including 1408 liver cancer cases and 1343 healthy controls. All patients with the diagnosis of liver cancer were confirmed by pathological examinations. Three studies used population-based (community populations) controls, while the other four studies used hospital-based controls. Overall, five studies were conducted in Asian populations, one study in a Caucasian population, and the other study was in an African population. All studies extracted DNA from peripheral blood except for two (Tanabe et al., 2008; Li et al., 2010). The classic polymerase chain reaction-restriction fragment length polymorphism (PCR-RFLP) method was performed in all studies. The HWE test was also conducted to evaluate the genotype distribution of the controls in all seven studies. The controls were found to be in HWE in all studies with respect to the polymorphisms selected (all P > 0.05). All quality scores of the studies included were higher than 20 (moderate-high quality). The characteristics and methodological quality of the studies included are summarized in Table 1.

\section{Meta-analysis results}

A summary of the meta-analysis findings of the association between the $E G F$ rs4444903 $(A>G)$ polymorphism and liver cancer risk is provided in Table 2. Heterogeneity 
was clearly present $(\mathrm{P}<0.05)$, which might have resulted from differences in ethnicity, source of controls, and detection samples; therefore, a random effects model was conducted to pool these results. The meta-analysis results revealed that the $\mathrm{G}$ variant of the rs 4444903 polymorphism was associated with an increased risk of liver cancer $(\mathrm{G}$ allele $v s$ A allele: $\mathrm{OR}=1.25$, $95 \% \mathrm{CI}=1.01-1.56, \mathrm{P}=0.040 ; \mathrm{GG}+\mathrm{AG} v s \mathrm{AA}: \mathrm{OR}=1.65,95 \% \mathrm{CI}=1.27-2.15, \mathrm{P}<0.001$; GG $v s$ AA: $\mathrm{OR}=1.77,95 \% \mathrm{CI}=1.34-2.35, \mathrm{P}<0.001$ ) (Figure 2). Results from subgroup analysis by ethnicity indicated that the $\mathrm{G}$ variant of rs4444903 the polymorphism may be a risk factor for liver cancer susceptibility among Asians $(\mathrm{GG}+\mathrm{AG} v s \mathrm{AA}$ : OR $=1.42,95 \% \mathrm{CI}$ $=1.04-1.94, \mathrm{P}=0.026$; GG vs $\mathrm{AA}: \mathrm{OR}=1.44,95 \% \mathrm{CI}=1.05-1.99, \mathrm{P}=0.025$ ) (Figure 3). Significant associations were also observed among Caucasians and Africans (all $\mathrm{P}<0.05$ ). In the subgroup analysis based on country, the $G$ variant of the rs 4444903 polymorphism showed significant associations with an increased risk of liver cancer in the China, USA, and Egypt subgroups under the dominant model (all $\mathrm{P}>0.05$ ). Further subgroup analyses by language, country, and detection sample indicated that the $\mathrm{G}$ variant of the rs 4444903 polymorphism may increase susceptibility to liver cancer in most subgroups (as shown in Table 2). Although no statistically significant association was found in the population-based subgroup (all $\mathrm{P}>$ $0.05)$, this result might lack sufficient reliability owing to estimation error from the effect size.

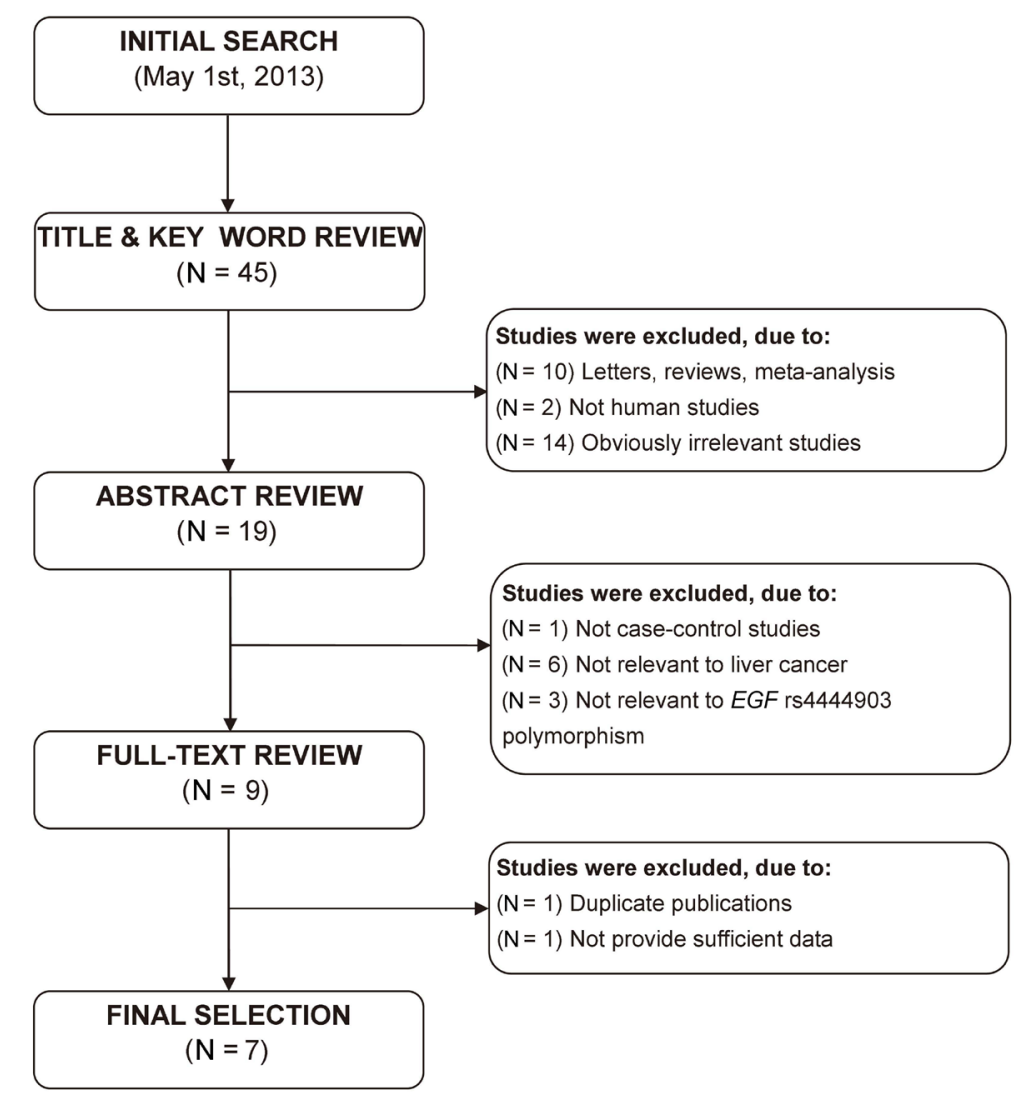

Figure 1. Flow chart showing study selection process. 


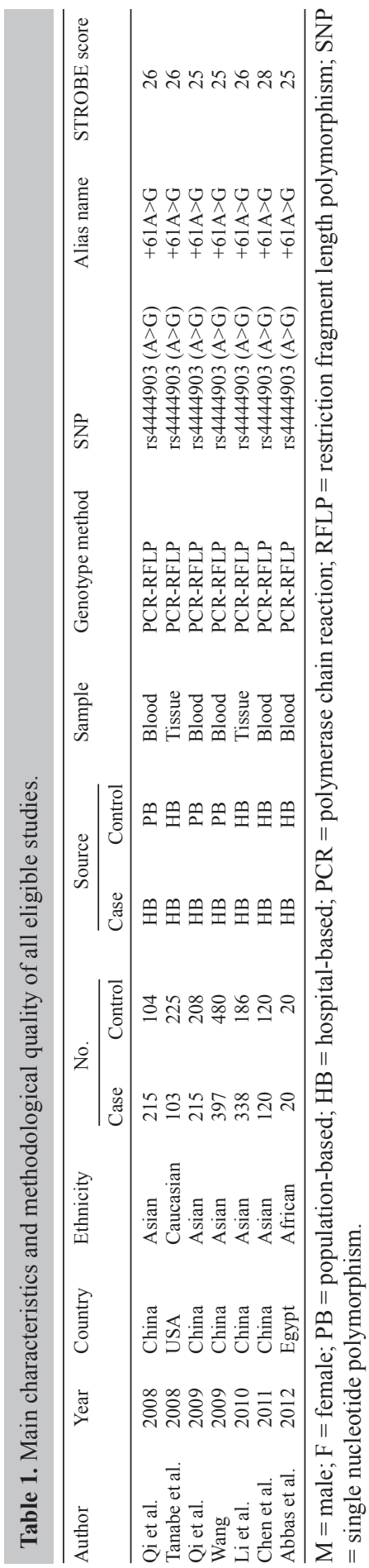


Y.L. Li et al.

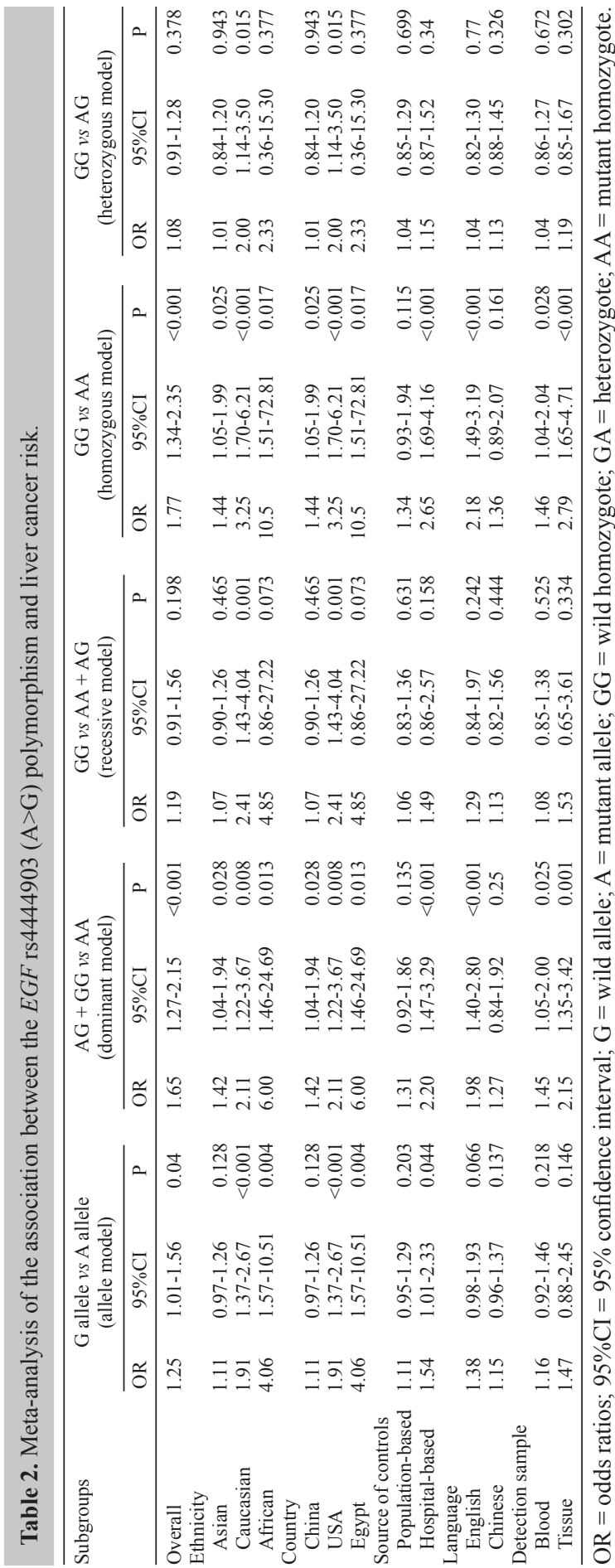




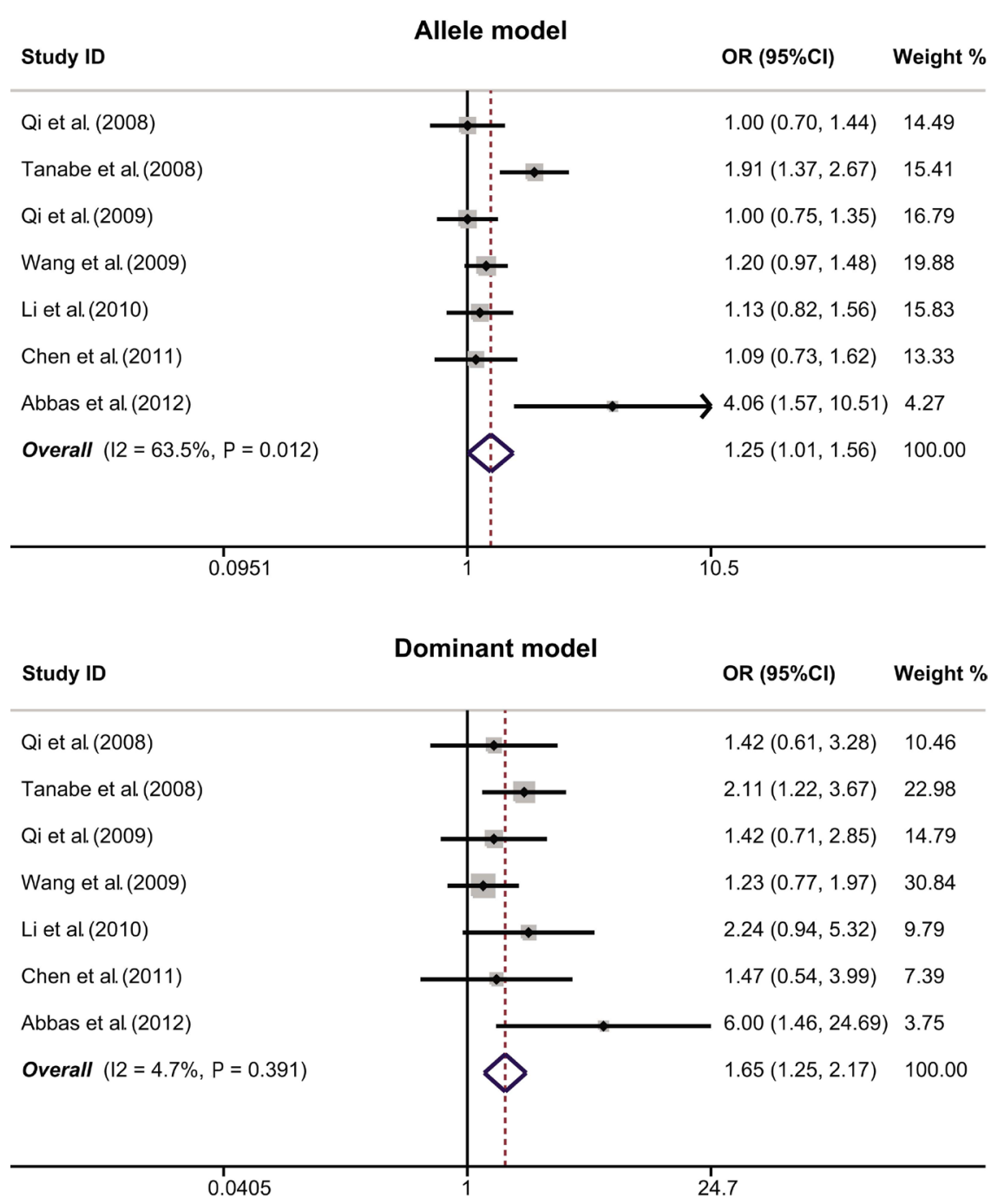

Figure 2. Forest plot for the association between the $E G F$ rs4444903 (A $>\mathrm{G})$ polymorphism and liver cancer risk under the allele and dominant models.

\section{Meta-regression and sensitivity analyses}

Univariate and multivariate meta-regression analyses were used to explore possible sources of heterogeneity among studies. The results showed that ethnicity could be a major source of heterogeneity as shown in Table 3. Sensitivity analysis was performed to assess the influence of each individual study on the pooled ORs by omitting individual studies consecutively. The results suggested that no individual study significantly affected the pooled OR of the association between the $E G F$ rs4444903 (A>G) polymorphism and liver cancer risk under the allele and dominant models, indicating that our results are statistically robust (Figure 4). 


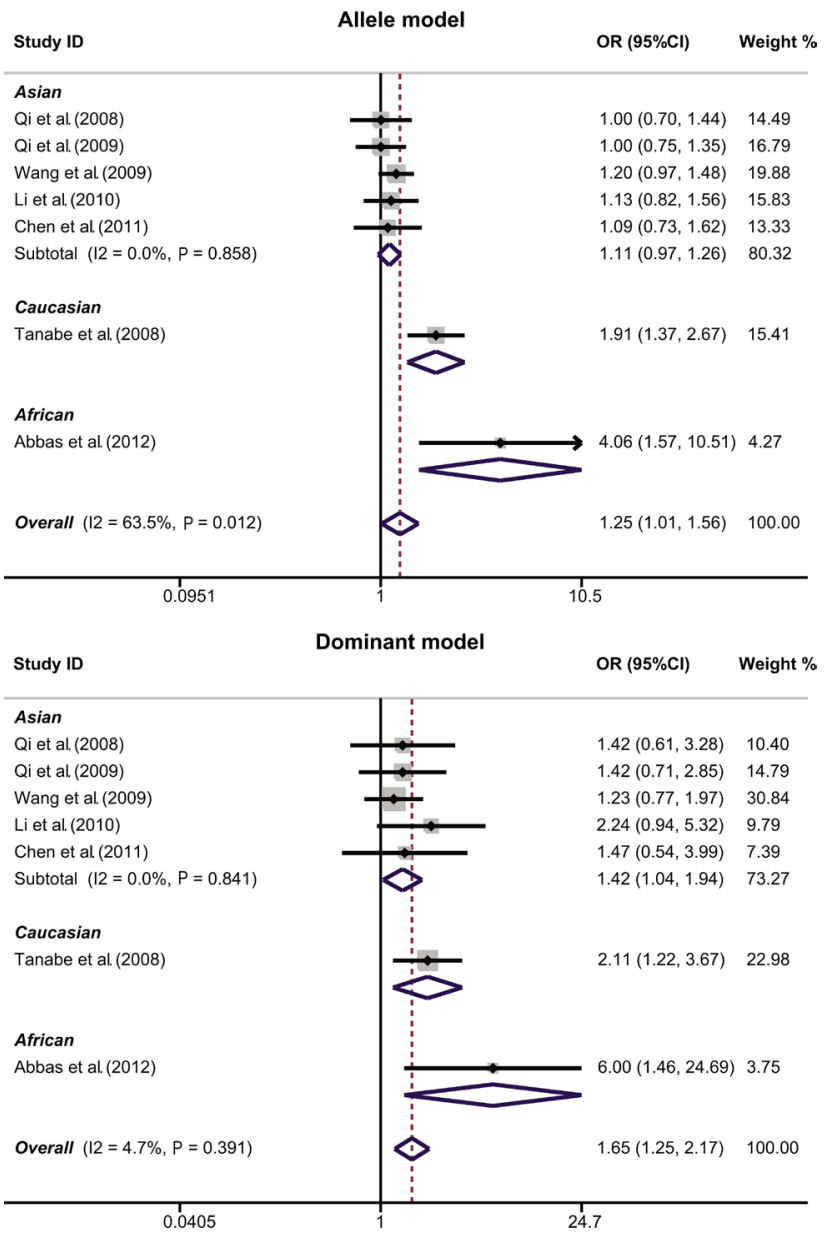

Figure 3. Subgroup analysis by ethnicity of the association between the $E G F$ rs 4444903 (A $>G)$ polymorphism and liver cancer risk under the allele and dominant models.

Table 3. Univariate and multivariate meta-regression analyses of potential sources of heterogeneity.

\begin{tabular}{|c|c|c|c|c|c|c|}
\hline \multirow[t]{2}{*}{ Heterogeneity factors } & \multirow[t]{2}{*}{ Coefficient } & \multirow[t]{2}{*}{ SE } & \multirow[t]{2}{*}{$\mathrm{Z}$} & \multirow[t]{2}{*}{$\mathrm{P}$} & \multicolumn{2}{|c|}{$95 \% \mathrm{CI}$} \\
\hline & & & & & LL & UL \\
\hline \multicolumn{7}{|l|}{ Publication year } \\
\hline Univariate & 0.123 & 0.143 & 0.86 & 0.391 & -0.158 & 0.403 \\
\hline Multivariate & -0.204 & 0.386 & -0.53 & 0.598 & -0.961 & 0.554 \\
\hline \multicolumn{7}{|l|}{ Ethnicity } \\
\hline Univariate & -0.536 & 0.307 & -1.75 & 0.080 & -1.137 & 0.064 \\
\hline Multivariate & 2.229 & 2.668 & 0.84 & 0.403 & -2.999 & 7.458 \\
\hline \multicolumn{7}{|l|}{ Country } \\
\hline Univariate & 0.535 & 0.256 & 2.10 & 0.036 & 0.035 & 1.037 \\
\hline Multivariate & 1.858 & 1.729 & 1.07 & 0.282 & -1.530 & 5.246 \\
\hline \multicolumn{7}{|l|}{ Source of control } \\
\hline Univariate & -0.516 & 0.273 & -1.89 & 0.059 & -1.054 & 0.020 \\
\hline Multivariate & -0.682 & 0.727 & -0.94 & 0.348 & -2.108 & 0.743 \\
\hline
\end{tabular}

$\mathrm{SE}=$ standard error; $95 \% \mathrm{CI}=95 \%$ confidence interval; $\mathrm{UL}=$ upper limit; $\mathrm{LL}=$ lower limit. 

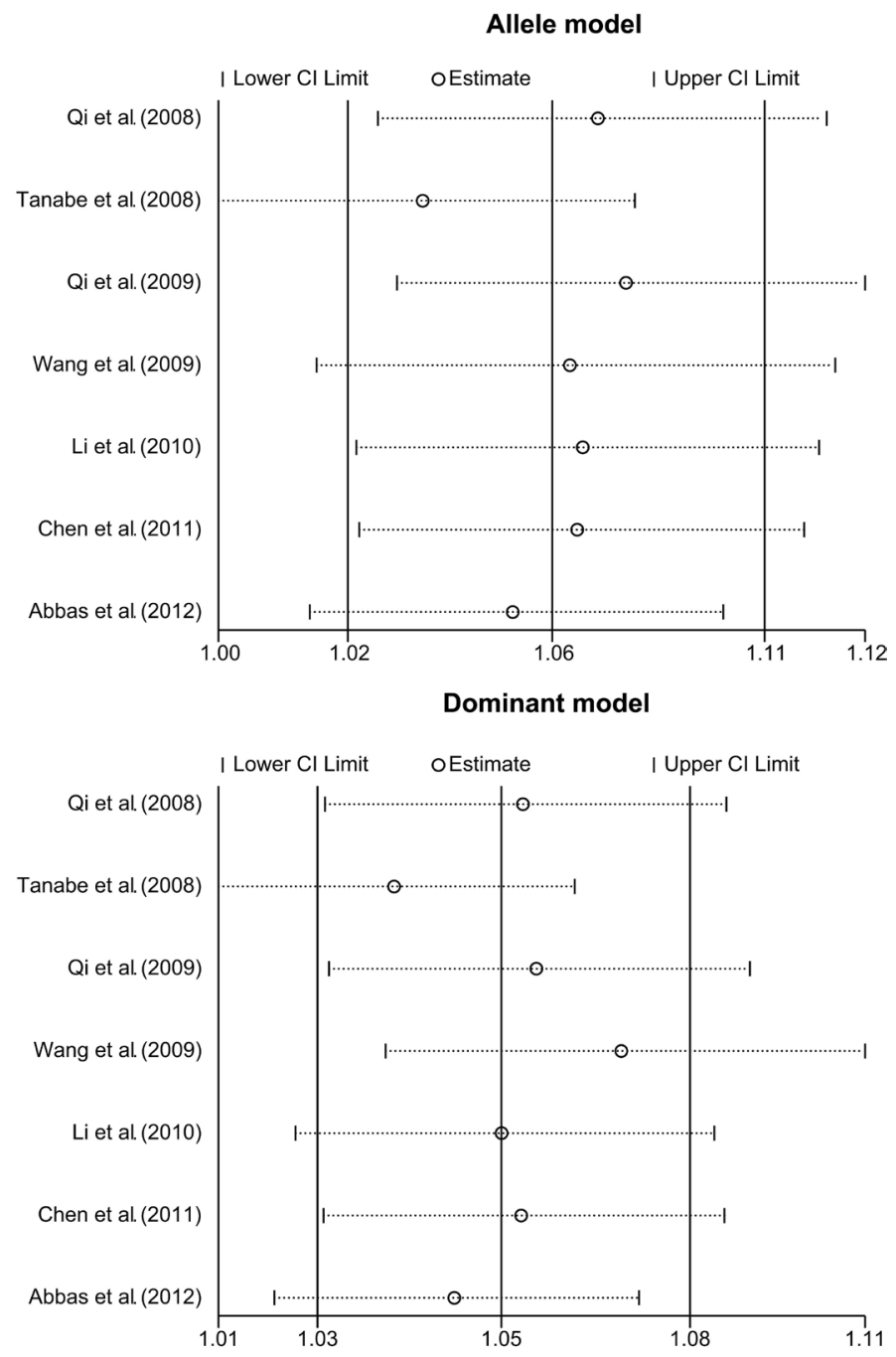

Figure 4. Sensitivity analyses for the association between the $E G F$ rs4444903 (A>G) polymorphism and liver cancer risk under the allele and dominant models. Results were computed by omitting each study in turn. Metaanalysis random-effects estimates (exponential form) were used. The two ends of the dotted lines represent the $95 \%$ confidence interval.

\section{Publication bias}

Begg's funnel plot and Egger's linear regression test were performed to assess the publication biases of the studies included. The shapes of the funnel plots did not reveal any evidence of obvious asymmetry under the allele and dominant models (Figure 5). The Egger test also did not display strong statistical evidence of publication bias (allele model: $\mathrm{P}=0.476$; dominant model: $\mathrm{P}=0.716$ ). 

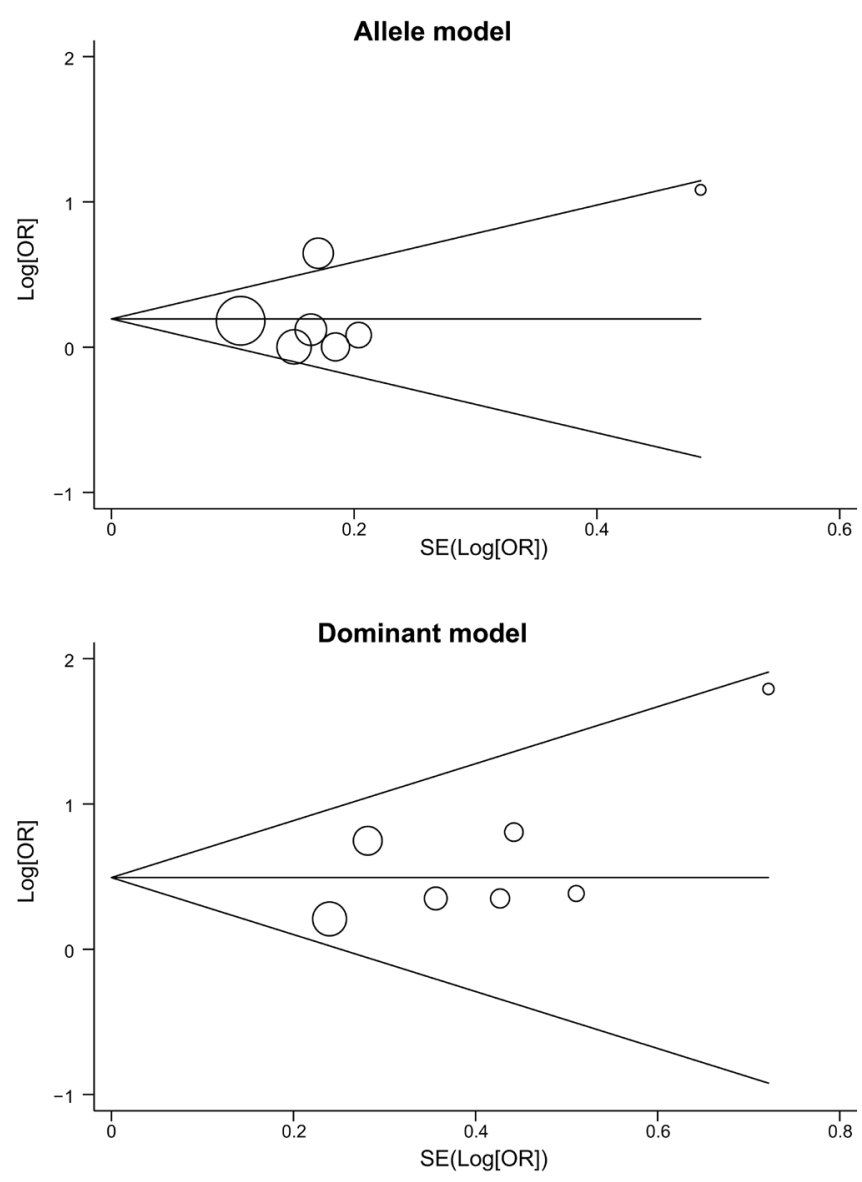

Figure 5. Begg's funnel plot for the association between the $E G F$ rs4444903 (A>G) polymorphism and liver cancer risk under the allele and dominant models. Each point represents a separate study for the association indicated. Log $[\mathrm{OR}]=$ natural logarithm of $\mathrm{OR} ; \mathrm{SE}=$ standard error. Horizontal line $=$ magnitude of the effect.

\section{DISCUSSION}

Epidermal growth factor, encoded by $E G F$, is an important molecule that plays a key role not only in cellular signaling, but also in several other cellular processes (de Mello et al., 2012; Del Vecchio et al., 2012). Epidermal growth factor can activate its high affinity cell surface receptor in certain signaling pathways, and has been implicated in the change of gene transcription, which can ultimately be attributed to the presence of tumors (Zhang et al., 2012). Recently, multiple studies have reported that the overexpression of epidermal growth factor is likely associated with risk of tumorigenesis in several human cancers, such as non-small cell lung cancer, colorectal cancer, and liver cancer (Chen et al., 2011; Daraei et al., 2012; de Mello et al., 2012). In addition, genetic evidence supports that functional polymorphisms induced by alternate splicing in the $5^{\prime}$ untranslated region of the $E G F$ gene affect serum epidermal growth factor levels (Qi et al., 2008; Tanabe et al., 2008; Wang, 2009). The 
human $E G F$ gene, located on chromosome 4q25-q27, is known as a crucial gene involved in the stimulation of proliferation, differentiation, and tumorigenesis of epidermal and epithelial tissues (Zhong et al., 2012). In recent years, numerous studies have suggested that the $E G F$ rs4444903 polymorphism, an A-to-G mutation at position 61 of the $5^{\prime}$ untranslated region, is associated with an increased risk of liver cancer (Qi et al., 2008; Abbas et al., 2012). Many genetic studies have investigated the association between the $E G F$ rs 4444903 polymorphism and liver cancer risk, but the results remain controversial (Qi et al., 2009; Xu et al., 2010; Chen et al., 2011; Zhong et al., 2012). This controversy could be explained by several factors, such as differences in study design, sample size, ethnicity, and source of controls, cancer types, and genotype methods.

To provide a comprehensive conclusion, we conducted the present meta-analysis of seven independent case-control studies, including 1408 liver cancer cases and 1343 healthy controls. The main results of this meta-analysis revealed that the $\mathrm{G}$ variant of the rs 4444903 polymorphism was associated with an increased risk of liver cancer, indicating that the $G$ variant may be a risk factor for liver cancer. Although the precise role of $E G F$ gene mutations in liver carcinogenesis remains unclear, a plausible mechanism could be that mutations of the $E G F$ gene might increase gene transcription after binding to its high-affinity cell surface receptor, which eventually causes overexpression of epidermal growth factor, thereby contributing to increased susceptibility to liver cancer. Since significant heterogeneity was observed, subgroup and meta-regression analyses were performed to explore potential sources of heterogeneity. Further subgroup analyses showed that the $E G F$ rs4444903 (A>G) polymorphism was associated with an increased risk of liver cancer in most subgroups, indicating that ethnicity, country, source of controls, language, and detection sample were not major sources of heterogeneity. However, meta-regression analyses indicated that ethnicity may be a major source of heterogeneity. These disparate results may be due to the small sample size resulting in substantial estimation errors. Our findings are consistent with those of previous studies suggesting that the $E G F$ rs4444903 (A>G) polymorphism may be associated with increased risk of liver cancer and may be useful biomarkers for predicting an individual's susceptibility to liver cancer.

Some limitations of this meta-analysis should be acknowledged. First, there were only seven articles included in the present meta-analysis; therefore, the sample size was relatively small and may not provide sufficient statistical power to estimate the correlation between the $E G F$ rs4444903 (A $>\mathrm{G})$ polymorphism and susceptibility to liver cancer. Therefore, more studies with larger sample size are needed to accurately provide a more representative statistical analysis. Second, as a type of a retrospective study, meta-analysis may encounter recall or selection bias, which could influence the reliability of our study results. Third, our lack of access to the original data from the studies limited further evaluations of the potential interactions between other factors and susceptibility to liver cancer, such as gene-environment and gene-gene interactions.

In summary, our meta-analysis indicated that the $G$ variant of the rs 4444903 polymorphism may increase the risk of liver cancer. The $E G F$ rs4444903 (A>G) polymorphism can be useful as a biomarker in predicting the development of liver cancer. Based on the limitations mentioned above, more detailed studies are needed to confirm our findings. Further studies are warranted to validate the association between $E G F$ gene polymorphisms with other gene polymorphisms and liver cancer risk. 


\section{REFERENCES}

Abbas E, Shaker O, Abd El AG, Ramadan H, et al. (2012). Epidermal growth factor gene polymorphism 61A/G in patients with chronic liver disease for early detection of hepatocellular carcinoma: a pilot study. Eur. J. Gastroenterol. Hepatol. 24: 458-463.

Altekruse SF, McGlynn KA, Dickie LA and Kleiner DE (2012). Hepatocellular carcinoma confirmation, treatment, and survival in surveillance, epidemiology, and end results registries, 1992-2008. Hepatology 55: 476-482.

Araujo AP, Costa BM, Pinto-Correia AL, Fragoso M, et al. (2011). Association between EGF $+61 \mathrm{~A} / \mathrm{G}$ polymorphism and gastric cancer in Caucasians. World J. Gastroenterol. 17: 488-492.

Chen K, Wei Y, Yang H and Li B (2011). Epidermal growth factor $+61 \mathrm{G} / \mathrm{A}$ polymorphism and the risk of hepatocellular carcinoma in a Chinese population. Genet. Test. Mol. Biomarkers 15: 251-255.

Chuang SC, La Vecchia C and Boffetta P (2009). Liver cancer: descriptive epidemiology and risk factors other than HBV and HCV infection. Cancer Lett. 286: 9-14.

da Costa BR, Cevallos M, Altman DG, Rutjes AW, et al. (2011). Uses and misuses of the STROBE statement: bibliographic study. BMJ Open 1: e000048.

Daraei A, Salehi R, Salehi M, Emami MH, et al. (2012). Effect of rs6983267 polymorphism in the 8q24 region and rs4444903 polymorphism in EGF gene on the risk of sporadic colorectal cancer in Iranian population. Med. Oncol. 29: 1044-1049.

de Mello RA, Ferreira M, Costa S, Costa BM, et al. (2012). Association between EGF +61 genetic polymorphisms and nonsmall cell lung cancer increased risk in a Portuguese population: a case-control study. Tumour. Biol. 33: 1341-1348.

Del Vecchio CA, Li G and Wong AJ (2012). Targeting EGF receptor variant III: tumor-specific peptide vaccination for malignant gliomas. Expert Rev. Vaccines 11: 133-144.

He JH, Li YM, Zhang QB, Ren ZJ, et al. (2011). Cyclooxygenase-2 promoter polymorphism -899G/C is associated with hepatitis B-related liver cancer in a Chinese population of Gansu Province. Chin. Med. J. 124: 4193-4197.

Higgins JP and Thompson SG (2002). Quantifying heterogeneity in a meta-analysis. Stat. Med. 21: 1539-1558.

Hu ZJ, Xue JF, Zhang XY, Shi XS, et al. (2010). Relationship between genetic polymorphism of ERCC1 and susceptibility to liver cancer. Zhonghua Liu Xing. Bing Xue Za Zhi 31: 1288-1291.

Hussain SP, Schwank J, Staib F, Wang XW, et al. (2007). TP53 mutations and hepatocellular carcinoma: insights into the etiology and pathogenesis of liver cancer. Oncogene 26: 2166-2176.

Ioannidis JP, Patsopoulos NA and Rothstein HR (2008). Reasons or excuses for avoiding meta-analysis in forest plots. BMJ 336: 1413-1415.

Kato S, Tajiri T, Matsukura N, Matsuda N, et al. (2003). Genetic polymorphisms of aldehyde dehydrogenase 2, cytochrome p450 2E1 for liver cancer risk in HCV antibody-positive japanese patients and the variations of CYP2E1 mRNA expression levels in the liver due to its polymorphism. Scand. J. Gastroenterol. 38: 886-893.

Kirk GD, Bah E and Montesano R (2006). Molecular epidemiology of human liver cancer: insights into etiology, pathogenesis and prevention from The Gambia, West Africa. Carcinogenesis 27: 2070-2082.

Kovar FM, Thallinger C, Marsik CL, Perkmann T, et al. (2009). The EGF 61A/G polymorphism - a predictive marker for recurrence of liver metastases from colorectal cancer. Wien. Klin. Wochenschr. 121: 638-643.

Li Y, Xie Q, Lu F, Zhao J, et al. (2010). Association between epidermal growth factor $61 \mathrm{~A} / \mathrm{G}$ polymorphism and hepatocellular carcinoma susceptibility in Chinese patients. Liver Int. 30: 112-118.

Peters JL, Sutton AJ, Jones DR, Abrams KR, et al. (2006). Comparison of two methods to detect publication bias in metaanalysis. JAMA 295: 676-680.

Qi P, Chen YM, Gao CF and Fang M (2008). Relationship between polymorphism of epidermal growth factor 5 'UTR variant G61A gene and hepatocellular carcinoma in patients with chronic hepatitis B virus infection in China. Chin. J. Biologicals 21: 1051-1053.

Qi P, Wang H, Chen YM, Sun XJ, et al. (2009). No association of EGF 5'UTR variant A61G and hepatocellular carcinoma in Chinese patients with chronic hepatitis B virus infection. Pathology 41: 555-560.

Srivatanakul P, Sriplung H and Deerasamee S (2004). Epidemiology of liver cancer: an overview. Asian Pac. J. Cancer Prev. 5: 118-125.

Tanabe KK, Lemoine A, Finkelstein DM, Kawasaki H, et al. (2008). Epidermal growth factor gene functional polymorphism and the risk of hepatocellular carcinoma in patients with cirrhosis. JAMA 299: 53-60.

Villanueva A, Forns X and Llovet JM (2012). Molecular epidemiology in HCV-related hepatocellular carcinoma: first steps. J. Hepatol. 57: 213-214.

Wang HX (2009). Epidermal Growth Factor Gene Polymorphisms Associated with Susceptibility to Hepatocellular Carcinoma [D]. Guangxi Medical Univeristy, Nanning. 1-55. 
Wang S, Zhang QY and Zhou RL (2012). Relationship between LAPTM4B gene polymorphism and susceptibility of primary liver cancer. Ann. Oncol. 23: 1864-1869.

Wu GY, Hasenberg T, Magdeburg R, Bonninghoff R, et al. (2009). Association between EGF, TGF-beta1, VEGF gene polymorphism and colorectal cancer. World J. Surg. 33: 124-129.

Xu W, Li Y, Wang X, Chen B, et al. (2010). Association between EGF promoter polymorphisms and cancer risk: a metaanalysis. Med. Oncol. 27: 1389-1397.

Yang Z, Wu Q, Shi Y, Nie Y, et al. (2012). Epidermal growth factor $61 \mathrm{~A}>\mathrm{G}$ polymorphism is associated with risk of hepatocellular carcinoma: a meta-analysis. Genet Test. Mol. Biomarkers 16: 1086-1091.

Yotsumoto F, Sanui A, Fukami T, Shirota K, et al. (2009). Efficacy of ligand-based targeting for the EGF system in cancer. Anticancer Res. 29: 4879-4885.

Zhang Z, Liu ZB, Ren WM, Ye XG, et al. (2012). The miR-200 family regulates the epithelial-mesenchymal transition induced by EGF/EGFR in anaplastic thyroid cancer cells. Int. J. Mol. Med. 30: 856-862.

Zhong JH, You XM, Gong WF, Ma L, et al. (2012). Epidermal growth factor gene polymorphism and risk of hepatocellular carcinoma: a meta-analysis. PLoS One 7: e32159.

Zintzaras E and Ioannidis JP (2005). Heterogeneity testing in meta-analysis of genome searches. Genet. Epidemiol. 28: 123-137. 